\title{
ABO Blood Group and RhD status Are Not Associated with Mortality and Morbidity in Critically III Patients; a Multicentre Observational Study of 29512 Patients
}

\author{
Thomas Kander ( $\nabla$ thomas.kander@med.lu.se ) \\ Lund University \\ Martin F. Bjurström \\ Lund University \\ Attila Frigyesi \\ Lund University \\ Magnus Jöud \\ Region Skåne \\ Caroline U. Nilsson \\ Lund University
}

\section{Research Article}

Keywords: ABO blood group, RhD blood group, Mortality, Morbidity, Blood group antigen, ABO blood group system, Epidemiology

Posted Date: January 11th, 2022

DOl: https://doi.org/10.21203/rs.3.rs-944620/v2

License: @ (i) This work is licensed under a Creative Commons Attribution 4.0 International License. Read Full License

Version of Record: A version of this preprint was published at BMC Anesthesiology on April 2nd, 2022. See the published version at https://doi.org/10.1186/s12871-022-01626-4. 


\section{Abstract}

Background. Previous studies have demonstrated an association between ABO blood groups and many types of disease. The present study primarily aimed to identify associations between $\mathrm{ABO}$ blood groups, RhD groups and mortality/morbidity outcomes in critically ill patients both in a main cohort and in six pre-defined subgroups. The secondary aim was to investigate any differences in transfusion requirement between the different $\mathrm{ABO}$ blood groups and RhD status.

Methods. Adult patients admitted to any of the five intensive care units (ICUs) in Skåne, Sweden, between February 2007 and April 2021 were eligible for inclusion. The outcomes were mortality analysed at 28- and 90-days as well as at the end of observation and morbidity measured using days alive and free of (DAF) invasive ventilation (DAF ventilation) and DAF circulatory support, including vasopressors or inotropes (DAF circulation), maximum Sequential Organ Failure Assessment score (SOFAmax) the first 28 days after admission and length of stay. All outcomes were analysed in separate multivariable regression models (adjusted for age and sex), generating odds or hazard ratios for each blood group and RhD status using blood group $\mathrm{O}$ and $\mathrm{RhD}$ negative as reference. Transfusion requirements were also investigated.

Results. In total, 29512 unique patients were included in the analyses. There were no significant differences for any of the outcomes between non-O blood groups and blood group 0, or between RhD groups. In five pre-defined subgroups (sepsis, septic shock, acute respiratory distress syndrome, cardiac arrest and trauma) there were no differences in mortality between non-0 blood groups and blood group $\mathrm{O}$ or between the RhD groups. The Covid-19 cohort was not investigated given the low number of patients. Furthermore, we could not demonstrate any differences in the number of transfused patients between the $A B O$ blood groups or between the RhD groups.

Conclusions. $\mathrm{ABO}$ blood type and $\mathrm{RhD}$ status do not appear to influence mortality or morbidity in a general critically ill patient population. There were no differences in the number of transfused patients between the ABO blood groups or between the RhD status groups.

\section{Background}

The ABO blood groups represent genetic traits that are expressed as antigens on the surface of erythrocytes and multiple other cell types, including intestinal mucosa, endothelium, kidney, and heart [1,2]. Previous studies have demonstrated an association between $\mathrm{ABO}$ blood groups and several different diseases [3]. For example, patients with non-O blood groups seem to be more susceptible to coronary artery disease and venous thrombosis as compared to blood group 0 , and blood group A can influence the risk of infectious disease [3,4]. Moreover, blood group 0 conveys a higher risk for bleeding complications, possibly due to observed lower circulating levels of von Willebrand factor (VWF) associated with this blood group [5]. Due to the emergence of the Coronavirus disease 2019 (Covid-19) pandemic, recent intense research efforts have been directed towards investigating ABO blood groups as putative risk factors for severe acute respiratory syndrome coronavirus-2 (SARS-CoV-2) disease. Although results are equivocal, blood group $\mathrm{O}$ appears to be protective against Covid-19 infection as compared to non- 0 blood groups, in particular blood group A. In addition to the decreased thrombotic tendency associated with blood group 0, protective effects may be mediated through, e.g., anti-A and anti-B antibodies [6-8]. Some data indicate that RhD status could impact risk for Covid-19 and diseases beyond those directly related to transfusion medicine and obstetrics [8], but RhD status has been far less studied in the critical care setting compared to the ABO blood groups.

Although many previous studies have demonstrated varying associations between ABO blood group affiliation and outcomes in subgroups of critically ill patients [9-20], to our knowledge, only one previous original study has examined a mixed critically ill patient population [21]. In a register-based study ( $n=7340)$, Slade et al. found that critically ill patients with blood group $A B$ exhibited a higher 90-day survival probability compared to patients with non-AB blood groups $(p=0.049$, unadjusted log-rank analysis) [21]. However, only short-term mortality was investigated and the analyses were not corrected for any confounding variables.

In an attempt to further investigate the influence of the $\mathrm{ABO}$ blood group on mortality and morbidity in a large cohort of mixed critically ill patients, we designed the current multicentre, register-based study. The primary aim was to identify any associations 
between the $\mathrm{ABO}$ blood groups, RhD status and mortality/morbidity both in the main cohort and in six pre-defined subgroups. The secondary aim was to investigate potential differences between the ABO blood groups and RhD status in transfusion requirement five days before admission and during the intensive care unit (ICU) stay.

\section{Methods}

\section{Study design and overview}

The study was approved by the National Ethical Review Board, Lund, Sweden, with registration numbers 2014/916 and 2018/866. As the study was exclusively observational the Board waived the requirement for informed consent. The manuscript was written in accordance with the STROBE statement for observational studies [22].

All patients $\geq 18$ years admitted to any of the five ICUs in southern Sweden (two at a university hospital (Skåne University Hospital, Lund and Malmö) and three at county hospitals (Helsingborg, Ystad and Kristianstad) having a total of 35 beds, between February 2007 and April 2021, were included. For patients with multiple ICU admissions, only the first admission was included. Patients without an established ABO blood group or RhD status and non-critically ill admitted patients, were excluded. Examples of non-critically ill admitted patients include patients admitted after elective surgery and patients admitted due to hospital shortage of general ward beds. Data were collected from the local quality register (PASIVA, Otimo Data AB, Kalmar, Sweden) and paired with data on $A B O$ blood groups, Rh status and transfusions, extracted from the laboratory information system (Flexlab/DoReMi, Tieto Sweden AB, Stockholm, Sweden) at Clinical Immunology and Transfusion Medicine, Region Skåne, Sweden.

\section{Outcomes}

Mortality was analysed at 28- and 90-days and at the end of observation (the point of data collection).

Morbidity was measured using days alive and free of (DAF) invasive ventilation (DAF ventilation) and DAF circulatory support, including vasopressors or inotropes (DAF circulation) the first 28 days after admission. As previously recommended, DAF was used without extra penalty for death [23]. Furthermore, the maximum Sequential Organ Failure Assessment-score (SOFA-max) during the ICU stay and the duration of the ICU stay itself ("Length of stay") were used to assess morbidity.

The number of patients that received red blood cells, plasma or platelets, five days prior to admission and during the ICU stay, were also recorded.

\section{Statistics}

The sample size was based on the number of available patients during the study period. Normality tests were used to test for normal distribution, and continuous variables were summarised with median (interquartile range) accordingly. Numbers were provided together with percentages.

All regression analyses were corrected for age and sex. All outcomes were analysed in separate multivariable regression models resulting in odds or hazard ratios for each $\mathrm{ABO}$ blood group and $\mathrm{RhD}$ status using blood group $\mathrm{O}$ and $\mathrm{RhD}$ negative as references. Mortality at 28 and 90 days after ICU admission were analysed using multivariable logistic regression. The HosmerLemeshow goodness-of-fit test was used to ensure correct calibration of the logistic regression models. The Cox regression hazard assumption was tested prior to the Cox-regression analyses for long-term mortality.

As the distribution of the DAF, the length-of-stay, and the SOFA-max variables did not fit any commonly used regression analyses, these variables were dichotomised. For the DAF variables, 24 hours of treatment was used as cut off, i.e., DAF $<27=1$ and $\mathrm{DAF} \geq 27=0$. For the length-of-stay and the SOFA-max variables, the cut off was applied at $1.5 \mathrm{ICU}$-days and 7 points, respectively, which corresponded to the median of both variables. All P-values $<0.05$ were considered significant. All analyses were performed using SPSS version 27 (SPSS Inc., Chicago, IL, USA). 


\section{Sensitivity analyses}

1) As previously described, $A B O$ blood groups and $R h D$ status may affect the severity of illness $[1,3,6,14]$. Hence, the primary analyses in the present study were not corrected for the severity of illness. However, in secondary sensitivity analyses, decided a priori, we tested if blood groups may add predictive value to the severity score Simplified Acute Physiology score 3 with estimated mortality risk (SAPS 3, EMR) by adding SAPS 3, EMR as an independent variable in the regression analyses.

2) Previous studies have demonstrated varying effects of $A B O$ blood groups and RhD status in different subgroups of critically ill patients [9-19]. In an attempt to further evaluate these effects, we divided the main cohort into six subgroups with the main diagnoses: sepsis, septic shock, acute respiratory distress syndrome (ARDS), Covid-19, cardiac arrest and trauma. The primary multivariable regression analyses were planned to be repeated for each subgroup.

\section{Results}

In total, 29512 unique patients were included in the analyses (Figure 1). Detailed baseline characteristics stratified according to ABO blood group and RhD status are shown in Table 1. In summary, baseline variables at admission were generally similar across the ABO blood groups, with the exceptions of age, heart rate and leucocyte count. There were no differences in baseline characteristics between the RhD status groups. For the whole cohort, the median age was 67 years (53-76), 58\% were men and the median SAPS 3, EMR recently calibrated for Swedish conditions was 18\% (6-40). 
Table 1

Baseline characteristics

\begin{tabular}{|c|c|c|c|c|c|c|c|c|c|c|}
\hline & Total & $\begin{array}{l}\text { Blgrp } \\
\text { A }\end{array}$ & $\begin{array}{l}\text { Blgrp } \\
\text { B }\end{array}$ & $\begin{array}{l}\text { Blgrp } \\
A B\end{array}$ & $\begin{array}{l}\text { Blgrp } \\
0\end{array}$ & $\begin{array}{l}P \text { - } \\
\text { value }\end{array}$ & \multicolumn{2}{|c|}{ RhD positive } & $\begin{array}{l}\text { RhD } \\
\text { negative }\end{array}$ & $\begin{array}{l}\mathrm{P} \text { - } \\
\text { value }\end{array}$ \\
\hline & $\begin{array}{l}n=29 \\
512\end{array}$ & $\begin{array}{l}n=12 \\
969\end{array}$ & $\begin{array}{l}n=3 \\
421\end{array}$ & $\begin{array}{l}n=1 \\
416\end{array}$ & $\begin{array}{l}n=11 \\
706\end{array}$ & & $\begin{array}{l}n=24 \\
797\end{array}$ & \multicolumn{3}{|c|}{$n=4715$} \\
\hline Blgrp, \% & & 44 & 12 & 4.7 & 40 & N.A. & 84 & \multicolumn{2}{|l|}{16} & N.A. \\
\hline $\begin{array}{l}\text { Blgrp in Sweden,\% } \\
\text { [24] }\end{array}$ & & 45 & 11 & 5.0 & 39 & N.A. & 82 & \multicolumn{2}{|l|}{18} & N.A. \\
\hline Age, years & $\begin{array}{l}67 \\
(53- \\
76)\end{array}$ & $\begin{array}{l}67 \\
(54- \\
76)\end{array}$ & $\begin{array}{l}66 \\
(51- \\
75)\end{array}$ & $\begin{array}{l}66 \\
(53- \\
75)\end{array}$ & $\begin{array}{l}68 \\
(54- \\
76)\end{array}$ & $<0.001$ & $\begin{array}{l}67(53 \\
-76)\end{array}$ & \multicolumn{2}{|c|}{$67(54-76)$} & 0.142 \\
\hline Male sex & $\begin{array}{l}16956 \\
(58)\end{array}$ & $\begin{array}{l}7406 \\
(57)\end{array}$ & $\begin{array}{l}1194 \\
(58)\end{array}$ & $\begin{array}{l}802 \\
(57)\end{array}$ & $\begin{array}{l}6754 \\
(58)\end{array}$ & 0.516 & $\begin{array}{l}14207 \\
(57)\end{array}$ & \multicolumn{2}{|c|}{$2749(58)$} & 0.198 \\
\hline SAPS 3 EMR, \% & $\begin{array}{l}18 \\
(5.8- \\
40)\end{array}$ & $\begin{array}{l}18 \\
(5.9- \\
40)\end{array}$ & $\begin{array}{l}18 \\
(5.8- \\
40)\end{array}$ & $\begin{array}{l}18 \\
(5.8- \\
38)\end{array}$ & $\begin{array}{l}19 \\
(5.8- \\
40)\end{array}$ & 0.762 & $\begin{array}{l}19 \\
(5.8- \\
40)\end{array}$ & \multicolumn{2}{|c|}{$18(5.8-38)$} & 0.255 \\
\hline \multicolumn{11}{|l|}{ Comorbidities } \\
\hline Cancer & $\begin{array}{l}2667 \\
(9.0)\end{array}$ & $\begin{array}{l}1204 \\
(9.2)\end{array}$ & $\begin{array}{l}292 \\
(8.5)\end{array}$ & $\begin{array}{l}114 \\
(8.1)\end{array}$ & $\begin{array}{l}1057 \\
(9.0)\end{array}$ & 0.591 & $\begin{array}{l}2246 \\
(9.1)\end{array}$ & \multicolumn{2}{|c|}{421 (8.9) } & 0.785 \\
\hline Heart failure & $\begin{array}{l}1714 \\
(5.8)\end{array}$ & $\begin{array}{l}708 \\
(5.4)\end{array}$ & $\begin{array}{l}186 \\
(5.4)\end{array}$ & $\begin{array}{l}99 \\
(7.0)\end{array}$ & $\begin{array}{l}721 \\
(6.2)\end{array}$ & 0.083 & $\begin{array}{l}1472 \\
(5.9)\end{array}$ & \multicolumn{2}{|c|}{$242(5.1)$} & 0.084 \\
\hline Blood malignancy & $\begin{array}{l}594 \\
(2.0)\end{array}$ & $\begin{array}{l}252 \\
(1.9)\end{array}$ & $\begin{array}{l}77 \\
(2.2)\end{array}$ & $\begin{array}{l}28 \\
(2.0)\end{array}$ & $\begin{array}{l}237 \\
((2.0)\end{array}$ & 0.858 & $\begin{array}{l}511 \\
(2.1)\end{array}$ & \multicolumn{2}{|c|}{$83(1.8)$} & 0.336 \\
\hline Cirrhosis & $\begin{array}{l}570 \\
(2.0)\end{array}$ & $\begin{array}{l}259 \\
(1.9)\end{array}$ & $\begin{array}{l}71 \\
(2.1)\end{array}$ & $\begin{array}{l}29 \\
(2.0)\end{array}$ & $\begin{array}{l}211 \\
(1.8)\end{array}$ & 0.803 & $\begin{array}{l}481 \\
(1.9)\end{array}$ & \multicolumn{2}{|c|}{$89(1.9)$} & 0.787 \\
\hline Immunosuppression & $\begin{array}{l}1432 \\
(4.9)\end{array}$ & $\begin{array}{l}660 \\
(5.1)\end{array}$ & $\begin{array}{l}158 \\
(4.6)\end{array}$ & $\begin{array}{l}59 \\
(4.2)\end{array}$ & $\begin{array}{l}555 \\
(4.7)\end{array}$ & 0.569 & $\begin{array}{l}1202 \\
(4.8)\end{array}$ & \multicolumn{2}{|c|}{$230(4.9)$} & 0.798 \\
\hline \multicolumn{11}{|l|}{ Admission route } \\
\hline Emergency dept. & $\begin{array}{l}13052 \\
(44)\end{array}$ & $\begin{array}{l}5704 \\
(44)\end{array}$ & $\begin{array}{l}1472 \\
(43)\end{array}$ & $\begin{array}{l}625 \\
(44)\end{array}$ & $\begin{array}{l}5251 \\
(45)\end{array}$ & 0.803 & $\begin{array}{l}10968 \\
(44)\end{array}$ & \multicolumn{2}{|c|}{$2084(44)$} & 0.981 \\
\hline Surgical theatre & $\begin{array}{l}3282 \\
(11)\end{array}$ & $\begin{array}{l}1451 \\
(11)\end{array}$ & $\begin{array}{l}405 \\
(12)\end{array}$ & $\begin{array}{l}171 \\
(12)\end{array}$ & $\begin{array}{l}1255 \\
(11)\end{array}$ & 0.757 & $\begin{array}{l}2777 \\
(11)\end{array}$ & \multicolumn{2}{|c|}{$505(11)$} & 0.888 \\
\hline General ward & $\begin{array}{l}9007 \\
(30)\end{array}$ & $\begin{array}{l}3992 \\
(31)\end{array}$ & $\begin{array}{l}1038 \\
(30)\end{array}$ & $\begin{array}{l}430 \\
(30)\end{array}$ & $\begin{array}{l}3547 \\
(30)\end{array}$ & 0.823 & $\begin{array}{l}7563 \\
(31)\end{array}$ & \multicolumn{2}{|c|}{$1444(31)$} & 0.787 \\
\hline Other ICU & $\begin{array}{l}1089 \\
(3.7)\end{array}$ & $\begin{array}{l}481 \\
(3.7)\end{array}$ & $\begin{array}{l}137 \\
(4.0)\end{array}$ & $\begin{array}{l}44 \\
(3.1)\end{array}$ & $\begin{array}{l}427 \\
(3.6)\end{array}$ & 0.110 & $\begin{array}{l}914 \\
(3.7)\end{array}$ & \multicolumn{2}{|c|}{$175(3.7)$} & 0.999 \\
\hline PACU & $\begin{array}{l}1076 \\
(3.6)\end{array}$ & $\begin{array}{l}469 \\
(3.6)\end{array}$ & $\begin{array}{l}120 \\
(3.5)\end{array}$ & $\begin{array}{l}47 \\
(3.3)\end{array}$ & $\begin{array}{l}440 \\
(3.8)\end{array}$ & 0.858 & $\begin{array}{l}899 \\
(3.6)\end{array}$ & \multicolumn{2}{|c|}{177 (3.7) } & 0.344 \\
\hline Missing data & $\begin{array}{l}2006 \\
(6.8)\end{array}$ & $\begin{array}{l}872 \\
(6.8)\end{array}$ & $\begin{array}{l}249 \\
(7.3)\end{array}$ & $\begin{array}{l}99 \\
(7.0)\end{array}$ & $\begin{array}{l}786 \\
(6.8)\end{array}$ & 0.686 & $\begin{array}{l}1686 \\
(6.8)\end{array}$ & \multicolumn{2}{|c|}{$319(6.8)$} & 0.877 \\
\hline \multicolumn{11}{|l|}{$\begin{array}{l}\text { Reason for } \\
\text { admission }\end{array}$} \\
\hline Sepsis & $\begin{array}{l}3016 \\
(10)\end{array}$ & $\begin{array}{l}1351 \\
(10)\end{array}$ & $\begin{array}{l}323 \\
(9.4)\end{array}$ & $\begin{array}{l}151 \\
(10.7)\end{array}$ & $\begin{array}{l}1191 \\
(10)\end{array}$ & 0.371 & $\begin{array}{l}2524 \\
(10)\end{array}$ & \multicolumn{2}{|c|}{$492(10)$} & 0.595 \\
\hline Septic shock & $\begin{array}{l}1366 \\
(4.6)\end{array}$ & $\begin{array}{l}626 \\
(4.8)\end{array}$ & $\begin{array}{l}132 \\
(3.9)\end{array}$ & $\begin{array}{l}60 \\
(4.2)\end{array}$ & $\begin{array}{l}548 \\
(4.7)\end{array}$ & 0.097 & $\begin{array}{l}1137 \\
(4.6)\end{array}$ & \multicolumn{2}{|c|}{$229(4.9)$} & 0.416 \\
\hline
\end{tabular}




\begin{tabular}{|c|c|c|c|c|c|c|c|c|c|c|}
\hline & Total & $\begin{array}{l}\text { Blgrp } \\
\text { A }\end{array}$ & $\begin{array}{l}\text { Blgrp } \\
\text { B }\end{array}$ & $\begin{array}{l}\text { Blgrp } \\
A B\end{array}$ & $\begin{array}{l}\text { Blgrp } \\
0\end{array}$ & $\begin{array}{l}\mathrm{P}- \\
\text { value }\end{array}$ & \multicolumn{2}{|c|}{ RhD positive } & $\begin{array}{l}\text { RhD } \\
\text { negative }\end{array}$ & $\begin{array}{l}\mathrm{P} \text { - } \\
\text { value }\end{array}$ \\
\hline ARDS & $\begin{array}{l}5642 \\
(19)\end{array}$ & $\begin{array}{l}2515 \\
(19)\end{array}$ & $\begin{array}{l}646 \\
(19)\end{array}$ & $\begin{array}{l}271 \\
(19)\end{array}$ & $\begin{array}{l}2210 \\
(19)\end{array}$ & 0.757 & $\begin{array}{l}4741 \\
(19)\end{array}$ & \multicolumn{2}{|c|}{$901(19)$} & 0.987 \\
\hline Covid-19 & $\begin{array}{l}338 \\
(1.1)\end{array}$ & $\begin{array}{l}157 \\
(1.2)\end{array}$ & $\begin{array}{l}45 \\
(1.3)\end{array}$ & $\begin{array}{l}18 \\
(1.3)\end{array}$ & $\begin{array}{l}122 \\
(1.0)\end{array}$ & 0.118 & $\begin{array}{l}295 \\
(1.2)\end{array}$ & \multicolumn{2}{|c|}{$43(0.9)$} & 0.100 \\
\hline Cardiac arrest & $\begin{array}{l}2562 \\
(8.7)\end{array}$ & $\begin{array}{l}1158 \\
(8.9)\end{array}$ & $\begin{array}{l}306 \\
(8.9)\end{array}$ & $\begin{array}{l}123 \\
(8.7)\end{array}$ & $\begin{array}{l}975 \\
(8.3)\end{array}$ & 0.371 & $\begin{array}{l}2138 \\
(8.6)\end{array}$ & \multicolumn{2}{|c|}{$424(9.0)$} & 0.407 \\
\hline Cardiovascular & $\begin{array}{l}8790 \\
(30)\end{array}$ & $\begin{array}{l}3837 \\
(30)\end{array}$ & $\begin{array}{l}1005 \\
(29)\end{array}$ & $\begin{array}{l}434 \\
(31)\end{array}$ & $\begin{array}{l}3514 \\
(30)\end{array}$ & 0.722 & $\begin{array}{l}7400 \\
((30)\end{array}$ & \multicolumn{2}{|c|}{$1390(30)$} & 0.618 \\
\hline Trauma & $\begin{array}{l}1583 \\
(5.4)\end{array}$ & $\begin{array}{l}700 \\
(5.4)\end{array}$ & $\begin{array}{l}189 \\
(5.5)\end{array}$ & $\begin{array}{l}66 \\
(4.7)\end{array}$ & $\begin{array}{l}628 \\
(5.4)\end{array}$ & 0.664 & $\begin{array}{l}1388 \\
(5.5)\end{array}$ & \multicolumn{2}{|c|}{$245(5.2)$} & 0.333 \\
\hline Hepatic & $\begin{array}{l}1520 \\
(5.2)\end{array}$ & $\begin{array}{l}673 \\
(5.2)\end{array}$ & $\begin{array}{l}187 \\
(5.4)\end{array}$ & $\begin{array}{l}68 \\
(4.8)\end{array}$ & $\begin{array}{l}592 \\
(5.1)\end{array}$ & 0.819 & $\begin{array}{l}1304 \\
(5.2)\end{array}$ & \multicolumn{2}{|c|}{$216(4.9)$} & 0.059 \\
\hline Abdominal & $\begin{array}{l}6674 \\
(23)\end{array}$ & $\begin{array}{l}2931 \\
(23)\end{array}$ & $\begin{array}{l}791 \\
(23)\end{array}$ & $\begin{array}{l}312 \\
(22)\end{array}$ & $\begin{array}{l}2640 \\
(23)\end{array}$ & 0.849 & $\begin{array}{l}5601 \\
(23)\end{array}$ & \multicolumn{2}{|c|}{$1073(23)$} & 0.798 \\
\hline CNS & $\begin{array}{l}11476 \\
(39)\end{array}$ & $\begin{array}{l}5054 \\
(39)\end{array}$ & $\begin{array}{l}1352 \\
(40)\end{array}$ & $\begin{array}{l}577 \\
(41)\end{array}$ & $\begin{array}{l}4493 \\
(38)\end{array}$ & 0.268 & $\begin{array}{l}9633 \\
(39)\end{array}$ & \multicolumn{2}{|c|}{1843 (39) } & 0.756 \\
\hline Renal & $\begin{array}{l}4868 \\
(16)\end{array}$ & $\begin{array}{l}2109 \\
(16)\end{array}$ & $\begin{array}{l}555 \\
(16)\end{array}$ & $\begin{array}{l}242 \\
(17)\end{array}$ & $\begin{array}{l}1962 \\
(17)\end{array}$ & 0.646 & $\begin{array}{l}4094 \\
(17)\end{array}$ & \multicolumn{2}{|c|}{$774(16)$} & 0.224 \\
\hline Metabolic & $\begin{array}{l}5023 \\
(17)\end{array}$ & $\begin{array}{l}2182 \\
(17)\end{array}$ & $\begin{array}{l}582 \\
(17)\end{array}$ & $\begin{array}{l}232 \\
(16)\end{array}$ & $\begin{array}{l}2027 \\
(17)\end{array}$ & 0.687 & $\begin{array}{l}4226 \\
(17)\end{array}$ & \multicolumn{2}{|c|}{797 (17) } & 0.816 \\
\hline $\begin{array}{l}\text { Days in hospital } \\
\text { before admission }\end{array}$ & $\begin{array}{l}0(0- \\
2)\end{array}$ & $\begin{array}{l}0(0- \\
2)\end{array}$ & $\begin{array}{l}0(0- \\
2)\end{array}$ & $\begin{array}{l}0(0- \\
2)\end{array}$ & $\begin{array}{l}0(0- \\
2)\end{array}$ & 0.675 & $\begin{array}{l}0(0- \\
2)\end{array}$ & \multicolumn{2}{|c|}{$0(0-2)$} & 0.491 \\
\hline \multicolumn{11}{|l|}{$\begin{array}{l}\text { Physiological } \\
\text { parameters }\end{array}$} \\
\hline Heart rate /min & $\begin{array}{l}100 \\
(80- \\
120)\end{array}$ & $\begin{array}{l}100 \\
(80- \\
120)\end{array}$ & $\begin{array}{l}100 \\
(80- \\
120)\end{array}$ & $\begin{array}{l}100 \\
(80- \\
120)\end{array}$ & $\begin{array}{l}100 \\
(80- \\
120)\end{array}$ & 0.014 & $\begin{array}{l}100 \\
(80- \\
120)\end{array}$ & \multicolumn{2}{|c|}{$100(80-118)$} & 0.518 \\
\hline $\mathrm{SBP}, \mathrm{mmHg}$ & $\begin{array}{l}100 \\
(80- \\
125)\end{array}$ & $\begin{array}{l}100 \\
(80- \\
125)\end{array}$ & $\begin{array}{l}100 \\
(80- \\
125)\end{array}$ & $\begin{array}{l}100 \\
(80- \\
125)\end{array}$ & $\begin{array}{l}100 \\
(80- \\
125)\end{array}$ & 0.755 & $\begin{array}{l}100 \\
(80- \\
125)\end{array}$ & \multicolumn{2}{|c|}{$101(80-125)$} & 0.661 \\
\hline Body temp., ${ }^{\circ} \mathrm{C}$ & $\begin{array}{l}37.0 \\
(36.2- \\
37.6)\end{array}$ & $\begin{array}{l}37.0 \\
(36.2- \\
37.6)\end{array}$ & $\begin{array}{l}37.0 \\
(36.3- \\
37.6)\end{array}$ & $\begin{array}{l}37.0 \\
(36.3- \\
37.6)\end{array}$ & $\begin{array}{l}37.0 \\
(36.2- \\
37.6)\end{array}$ & 0.658 & $\begin{array}{l}37.0 \\
(36.2- \\
37.6)\end{array}$ & \multicolumn{2}{|c|}{$\begin{array}{l}37.0(36.2- \\
37.7)\end{array}$} & 0.846 \\
\hline Leucocytes, $\times 10^{9} / \mathrm{L}$ & $\begin{array}{l}12 \\
(8.8- \\
17)\end{array}$ & $\begin{array}{l}12 \\
(8.7- \\
17)\end{array}$ & $\begin{array}{l}12 \\
(8.6- \\
18)\end{array}$ & $\begin{array}{l}13 \\
(8.8- \\
18)\end{array}$ & $\begin{array}{l}13 \\
(8.9- \\
18)\end{array}$ & 0.031 & $\begin{array}{l}12 \\
(8.7- \\
17)\end{array}$ & \multicolumn{2}{|c|}{$13(8.9-18)$} & 0.102 \\
\hline Creatinine, $\mu \mathrm{mol} / \mathrm{L}$ & $\begin{array}{l}94 \\
(68- \\
145)\end{array}$ & $\begin{array}{l}94 \\
(68- \\
143)\end{array}$ & $\begin{array}{l}94 \\
(70- \\
145)\end{array}$ & $\begin{array}{l}92 \\
(68- \\
146)\end{array}$ & $\begin{array}{l}95 \\
(68- \\
147)\end{array}$ & 0.604 & $\begin{array}{l}94 \\
(68- \\
145)\end{array}$ & \multicolumn{2}{|c|}{$94(70-147)$} & 0.374 \\
\hline Bilirubin, $\mu \mathrm{mol} / \mathrm{L}$ & $\begin{array}{l}10 \\
(6.0- \\
18)\end{array}$ & $\begin{array}{l}10 \\
(6.0- \\
17)\end{array}$ & $\begin{array}{l}10 \\
(6.0- \\
18)\end{array}$ & $\begin{array}{l}10 \\
(6.0- \\
18)\end{array}$ & $\begin{array}{l}10 \\
(6.0- \\
18)\end{array}$ & 0.241 & $\begin{array}{l}10 \\
(6.0- \\
18)\end{array}$ & \multicolumn{2}{|c|}{$11(6.2-18)$} & 0116 \\
\hline $\begin{array}{l}\text { Platelet count, } \\
\times 10^{9} / \mathrm{L}\end{array}$ & $\begin{array}{l}211 \\
(151- \\
280)\end{array}$ & $\begin{array}{l}212 \\
(152- \\
280)\end{array}$ & $\begin{array}{l}208 \\
(147- \\
275)\end{array}$ & $\begin{array}{l}213 \\
(153- \\
281)\end{array}$ & $\begin{array}{l}211 \\
(151- \\
280)\end{array}$ & 0.368 & $\begin{array}{l}212 \\
(151- \\
280)\end{array}$ & \multicolumn{2}{|c|}{$\begin{array}{l}207(148- \\
277)\end{array}$} & 0.069 \\
\hline Blood pH & $\begin{array}{l}7.34 \\
(7.25- \\
7.41)\end{array}$ & $\begin{array}{l}7.34 \\
(7.25- \\
7.41)\end{array}$ & $\begin{array}{l}7.34 \\
(7.25- \\
7.41)\end{array}$ & $\begin{array}{l}7.34 \\
(7.24- \\
7.41)\end{array}$ & $\begin{array}{l}7.34 \\
(7.25- \\
7.41)\end{array}$ & 0.650 & $\begin{array}{l}7.34 \\
(7.25- \\
7.41)\end{array}$ & \multicolumn{2}{|c|}{$\begin{array}{l}7.34(7.25- \\
7.41)\end{array}$} & 0.793 \\
\hline
\end{tabular}


No imputations of missing data were performed. All baseline variables had $<10 \%$ missing data.

\section{Outcomes}

Descriptive values for the outcomes are summarised in Table 2. There were no differences between the ABO blood groups or the $\mathrm{RhD}$ groups in these analyses.

Table 2

Outcomes

\begin{tabular}{|c|c|c|c|c|c|c|c|c|c|}
\hline & Total & Blgrp A & Blgrp B & Blgrp AB & Blgrp 0 & $\begin{array}{l}\mathrm{P}- \\
\text { value }\end{array}$ & $\begin{array}{l}\text { RhD } \\
\text { positive }\end{array}$ & $\begin{array}{l}\text { RhD } \\
\text { negative }\end{array}$ & $\begin{array}{l}\mathrm{P} \text { - } \\
\text { value }\end{array}$ \\
\hline & $n=29512$ & $n=12969$ & $\mathrm{n}=3421$ & $n=1416$ & $n=11706$ & & $\begin{array}{l}n=24 \\
797\end{array}$ & $n=4715$ & \\
\hline $\begin{array}{l}\text { 28-day } \\
\text { mortality }\end{array}$ & $\begin{array}{l}7169 \\
(24)\end{array}$ & $\begin{array}{l}3153 \\
(24)\end{array}$ & 795 (23) & $353(25)$ & $\begin{array}{l}2868 \\
(25)\end{array}$ & 0.448 & $\begin{array}{l}6044 \\
(25)\end{array}$ & $\begin{array}{l}1125 \\
(24)\end{array}$ & 0.451 \\
\hline $\begin{array}{l}90-\text { day } \\
\text { mortality }\end{array}$ & $\begin{array}{l}8675 \\
(29)\end{array}$ & $\begin{array}{l}3828 \\
(29)\end{array}$ & 958 (28) & 432 (30) & $\begin{array}{l}3457 \\
(29)\end{array}$ & 0.237 & $\begin{array}{l}7303 \\
(29)\end{array}$ & $\begin{array}{l}1372 \\
(29)\end{array}$ & 0.626 \\
\hline DAFvent & $\begin{array}{l}27(17- \\
28)\end{array}$ & $\begin{array}{l}27(16- \\
28)\end{array}$ & $\begin{array}{l}27(19- \\
28)\end{array}$ & $\begin{array}{l}27(17- \\
28)\end{array}$ & $\begin{array}{l}27(16- \\
28)\end{array}$ & 0.774 & $\begin{array}{l}27(17- \\
28)\end{array}$ & $\begin{array}{l}27(17- \\
28)\end{array}$ & 0.188 \\
\hline DAFcirc & $\begin{array}{l}28(22- \\
28)\end{array}$ & $\begin{array}{l}28(22- \\
28)\end{array}$ & $\begin{array}{l}28(23- \\
28)\end{array}$ & $\begin{array}{l}28(22- \\
28)\end{array}$ & $\begin{array}{l}28(21- \\
28)\end{array}$ & 0.356 & $\begin{array}{l}28(22- \\
28)\end{array}$ & $\begin{array}{l}28(22- \\
28)\end{array}$ & 0.608 \\
\hline SOFA-max & $7(4-10)$ & $7(4-10)$ & $7(4-10)$ & $7(4-10)$ & $7(4-10)$ & 0.867 & $7(4-10)$ & $7(4-10)$ & 0.813 \\
\hline $\begin{array}{l}\text { Length-of-stay, } \\
\text { days }\end{array}$ & $\begin{array}{l}1.5(0.7- \\
3.4)\end{array}$ & $\begin{array}{l}1.5(0.7- \\
3.3)\end{array}$ & $\begin{array}{l}1.5(0.7- \\
3.5)\end{array}$ & $\begin{array}{l}1.6(0.8- \\
3.5)\end{array}$ & $\begin{array}{l}1.5(0.7- \\
3.3)\end{array}$ & 0.154 & $\begin{array}{l}1.5(0.7- \\
3.4)\end{array}$ & $\begin{array}{l}1.5(0.7- \\
3.3)\end{array}$ & 0.646 \\
\hline
\end{tabular}

Detailed results of the multivariable analyses are presented in Table 3. The goodness of fit test was not significant for any of the models. The proportional hazards assumption was not violated for or any of the models $(P>0.05)$. In summary, there were no differences for any of the outcomes between non-O blood groups and blood group 0 , or between RhD groups. In the first set of sensitivity analyses, including SAPS 3 as an independent variable, there were no differences in any of the mortality/morbidity outcomes between non-0 blood groups and blood group 0, or between RhD status groups (Table 4). SAPS 3 was strongly associated with all outcomes. In the second set of sensitivity analyses, where the main cohort was divided into six pre-defined subgroups, the lack of associations between the mortality/morbidity outcomes and the ABO blood group and RhD status remained largely unchanged in five of the subgroups, as compared to the main analyses. The Covid-19 cohort was not analysed given the low number of patients. Nevertheless, in the subgroups septic shock and cardiac arrest, blood group AB was associated with a longer length-of-stay as compared to blood group O. In the ARDS-subgroup, blood group A was associated with DAF ventilation. There were no differences in mortality variables between non-O blood groups and blood group 0 , or between RhD status, in any of the subgroups. Further details are provided in Additional file 1. 
Table 3

Multivariable regression analyses with $95 \%$ confidence interval for odds or hazard ratio

\begin{tabular}{|lllll|}
\hline & Blgrp A & Blgrp B & Blgrp AB & RhD positive \\
\hline Mortality & & & & \\
\hline 28-day mortality & $0.940-1.060$ & $0.902-1.086$ & $0.936-1.218$ & $0.970-1.127$ \\
\hline 90-day mortality & $0.954-1.069$ & $0.904-1.078$ & $0.970-1.247$ & $0.964-1.112$ \\
\hline Long-term mortality & $0.987-1.056$ & $0.945-1.049$ & $0.982-1.141$ & $0.985-1.075$ \\
\hline Morbidity & & & & \\
\hline DAFvent & $0.987-1.095$ & $0.946-1.108$ & $0.937-1.177$ & $1.000-1.137$ \\
\hline DAFcirc & $0.929-1.033$ & $0.964-1.134$ & $0.984-1.243$ & $0.967-1.105$ \\
\hline SOFA-max & $0.953-1.063$ & $0.961-1.135$ & $0.932-1.186$ & $0.959-1.099$ \\
\hline Length-of-stay & $0.953-1.054$ & $0.976-1.138$ & $0.968-1.209$ & $0.956-1.083$ \\
\hline
\end{tabular}

Table 4

First set of sensitivity analyses. Multivariable regression analyses with $95 \%$ confidence interval for odds or hazard ratio

\begin{tabular}{|llllll|}
\hline & SAPS3, EMR & Blgrp A & Blgrp B & Blgrp AB & RhD positive \\
\hline Mortality & & & & & \\
\hline 28-day mortality & $1.045-1.048^{\star}$ & $0.930-1.067$ & $0.873-1.080$ & $0.910-1.231$ & $0.937-1.114$ \\
\hline 90-day mortality & $1.043-1.046^{\star}$ & $0.946-1.076$ & $0.875-1.068$ & $0.944-1.255$ & $0.933-1.097$ \\
\hline Long-term mortality & $1.023-1.024^{\star}$ & $0.988-1.059$ & $0.931-1.038$ & $0.941-1.100$ & $0.958-1.046$ \\
\hline Morbidity & & & & & \\
\hline DAFvent & $1.048-1.051^{\star}$ & $0.998-1.124$ & $0.941-1.129$ & $0.930-1.208$ & $0.971-1.126$ \\
\hline DAFcirc & $1.041-1.044^{\star}$ & $0.917-1.033$ & $0.955-1.146$ & $0.975-1.267$ & $0.931-1.080$ \\
\hline SOFA-max & $1.063-1.067^{\star}$ & $0.953-1.086$ & $0.940-1.148$ & $0.926-1.236$ & $0.907-1.068$ \\
\hline Length-of-stay & $1.011-1.014 *$ & $0.958-1.063$ & $0.982-1.153$ & $0.974-1.228$ & $0.959-1.093$ \\
\hline
\end{tabular}




\section{Table 5}

Number of patients transfused

\begin{tabular}{|c|c|c|c|c|c|c|c|c|c|}
\hline & Total & Blgrp A & Blgrp B & $\begin{array}{l}\text { Blgrp } \\
A B\end{array}$ & Blgrp 0 & $\begin{array}{l}P \text { - } \\
\text { value }\end{array}$ & $\begin{array}{l}\text { RhD } \\
\text { positive }\end{array}$ & $\begin{array}{l}\text { RhD } \\
\text { negative }\end{array}$ & $\begin{array}{l}\text { P- } \\
\text { value }\end{array}$ \\
\hline & $\begin{array}{l}n=29 \\
512\end{array}$ & $\begin{array}{l}n=12 \\
969\end{array}$ & $\begin{array}{l}n=3 \\
421\end{array}$ & $\begin{array}{l}n=1 \\
416\end{array}$ & $\begin{array}{l}n=11 \\
706\end{array}$ & & $\begin{array}{l}n=24 \\
797\end{array}$ & $n=4715$ & \\
\hline \multicolumn{10}{|l|}{$\begin{array}{l}\text { Five days prior to } \\
\text { admission }\end{array}$} \\
\hline Red blood cells & $\begin{array}{l}2713 \\
(9.2)\end{array}$ & $\begin{array}{l}1202 \\
(9.3)\end{array}$ & $\begin{array}{l}313 \\
(9.2)\end{array}$ & $\begin{array}{l}124 \\
(8.8)\end{array}$ & $\begin{array}{l}1074 \\
(9.2)\end{array}$ & 0.942 & $\begin{array}{l}2287 \\
(9.2)\end{array}$ & $426(9.0)$ & 0.674 \\
\hline Plasma & $\begin{array}{l}705 \\
(2.4)\end{array}$ & $\begin{array}{l}291 \\
(2.2)\end{array}$ & $\begin{array}{l}94 \\
(2.8)\end{array}$ & $\begin{array}{l}28 \\
(2.0)\end{array}$ & $\begin{array}{l}705 \\
(2.4)\end{array}$ & 0.197 & $600(2.4)$ & $105(2.2)$ & 0.424 \\
\hline Platelets & $\begin{array}{l}425 \\
(1.4)\end{array}$ & $\begin{array}{l}183 \\
(1.4)\end{array}$ & $\begin{array}{l}60 \\
(1.8)\end{array}$ & $\begin{array}{l}19 \\
(1.3)\end{array}$ & $\begin{array}{l}163 \\
(1.4)\end{array}$ & 0.430 & 367 (1.5) & $58(1.2)$ & 0.185 \\
\hline \multicolumn{10}{|l|}{ During ICU-stay } \\
\hline Red blood cells & $\begin{array}{l}11344 \\
(38)\end{array}$ & $\begin{array}{l}4968 \\
(38)\end{array}$ & $\begin{array}{l}1321 \\
(39)\end{array}$ & $\begin{array}{l}545 \\
(39)\end{array}$ & $\begin{array}{l}4510 \\
(39)\end{array}$ & 0.971 & $\begin{array}{l}9534 \\
(38)\end{array}$ & $\begin{array}{l}1810 \\
(38)\end{array}$ & 0.915 \\
\hline Plasma & $\begin{array}{l}5385 \\
(18)\end{array}$ & $\begin{array}{l}2395 \\
(18)\end{array}$ & $\begin{array}{l}626 \\
(18)\end{array}$ & $\begin{array}{l}238 \\
(17)\end{array}$ & $\begin{array}{l}2128 \\
(18)\end{array}$ & 0.507 & $\begin{array}{l}4536 \\
(18)\end{array}$ & $848(18)$ & 0.604 \\
\hline Platelets & $\begin{array}{l}2840 \\
(9.6)\end{array}$ & $\begin{array}{l}1297 \\
(10)\end{array}$ & $\begin{array}{l}353 \\
(10)\end{array}$ & $\begin{array}{l}120 \\
(8.5)\end{array}$ & $\begin{array}{l}1070 \\
(9.2)\end{array}$ & 0.056 & $\begin{array}{l}2409 \\
(9.7)\end{array}$ & $431(9.2)$ & 0.216 \\
\hline
\end{tabular}

\section{Transfusions}

In total, $38 \%$ of the patients were transfused with red blood cells, $18 \%$ with plasma and $9.6 \%$ with platelets during the ICU stay (Table 5). There were no differences between the ABO blood groups or the RhD groups, neither in the number of patients transfused five days prior to the admission nor during the ICU stay.

\section{Discussion}

In this large multicentre observational study, we have demonstrated that neither the ABO blood group nor the RhD status is associated with mortality or morbidity in a mixed critically ill patient population. In sensitivity analyses, the ABO blood group and the RhD status did not add predictive value to the SAPS 3, and in five different subgroup analyses there were no differences in mortality between non-O blood groups and blood group $\mathrm{O}$ or between the RhD status. Furthermore, we could not demonstrate any differences in the number of transfused patients between the ABO blood groups or between the RhD status.

Based on previous findings, the ABO blood group is likely to affect homeostasis through several mechanisms [1-3]. However, our results imply that this effect is subtle, if not irrelevant, in a large mixed cohort of critically ill patients. It is possible that the ABO blood group may influence outcomes in certain clinical populations suffering from specific diseases; nevertheless, in contrast to a number of previous reports, we did not observe a mortality effect in any of the subgroups (sepsis, septic shock, ARDS, Covid-19, cardiac arrest and trauma). However, it should be noted that in the subgroups septic shock and cardiac arrest, blood group $A B$ was associated with a longer length-of-stay as compared to blood group O. In the ARDS-subgroup, blood group A was associated with increased ventilator time as measured with DAF ventilation.

In contrast to the absence of associations between the $\mathrm{ABO}$ blood group and outcomes in the present study of mixed critically ill patients, Slade et al. found that blood group AB conferred a 90-day mortality benefit compared to other ABO blood groups in a similar but smaller patient cohort in the United Kingdom [21]. However, it should be noted that only $3 \%$ of patients had blood 
group $A B$, and although baseline data were similar for the different $A B O$ blood groups, the mortality analyses were unadjusted, which may at least in part explain the differences compared to our results.

Recent studies have largely focused on Covid-19, where meta-analytic data indicate that blood group 0 might be protective against Covid-19, whereas blood group $A$ is associated with an increased risk of infection [7]. Apart from the lower thrombotic risk associated with blood group 0 , anti-A has also been reported to block virus entry into cells through interaction with the receptor angiotensin-converting enzyme-2 [8]. Unfortunately, the number of patients in the Covid-19 subgroup, $n=338$, in the present study was deemed too small to analyse.

The influence of ABO-blood groups has also been investigated in critically ill patients with non-Covid-19 disease, such as sepsis and ARDS. In two separate studies, Reilly et al. demonstrated that blood group A is associated with increased risk for ARDS in sepsis (and trauma), possibly due to dysfunction of endothelium and microvasculature, as indicated by altered levels of biomarkers (e.g., soluble thrombomodulin and selectins) $[14,15]$. These findings are supported by results from a large study of critically ill sepsis patients evaluating $A B O$ blood groups and biomarkers of endothelial damage, which demonstrated moderately decreased mortality for blood group B [12]. In the present study, the potential effect of the ABO-blood group did not influence morbidity and mortality outcomes for any of the sepsis ( $n=3$ 016), septic shock ( $n=1366)$ or ARDS ( $n=5$ 642) subgroups.

The increased risk of bleeding for patients with blood group 0 is mainly mediated by lower vWF levels (and associated lower factor VIII levels) [5]. This was not reflected in increased transfusion requirements in the present study. Blood group 0 has been associated with worse outcomes in severe trauma and increased transfusion volume in severe abdominal trauma, which could be due to the proposed haemostatic effects $[17,18]$. As mentioned above, Reilly et al. found a connection between blood group $A$ and the risk of ARDS in trauma patients $[14,15]$. Here, congruent with findings from two previous studies, we did not observe worse outcomes for blood group 0 in the trauma subgroup $[9,10]$.

The Rh blood group antigen RhD is routinely analysed together with the ABO blood group. RhD blood group is less studied than the $A B O$ system for its association with diseases, including critical illness. We found no effect of RhD status on mortality or morbidity in the general critically ill population or in any specific subgroup. The RhD antigen is only expressed on red blood cells and is primarily of clinical interest in the context of haemolytic reactions (of the newborn and in case of blood transfusion). Individuals who are RhD-negative may have some protection against Covid-19 infection, but the pathophysiological basis is unclear [8].

Although an individual cannot change $\mathrm{ABO}$ blood group or RhD status, except in the rare case of stem cell transplantation, the results of the present study expand important knowledge regarding associations between ABO groups, RhD status, mortality and morbidity. In addition to epidemiological studies, such as the present work, studies investigating pathophysiological alterations associated with blood groups, e.g., changes in vWF levels, blockage of virus-receptors and endotheliopathy, should be given high priority since detailed mechanistic insights may potentially guide development of future personalised therapies.

\section{Limitations and strengths}

We recognise the limitations in the present study given its retrospective nature, including the absence of a published study protocol. Secondly, data on ethnicity is missing. Thirdly, the division into subgroups in the second set of sensitivity analyses was performed based on diagnoses coded by the treating physician. Hence it cannot be ruled out that some diagnoses were missed. Fourthly, the Covid-19 cohort was deemed too small for robust analyses of independent risk factors. Strengths of the study include a larger sample size compared to previous similar studies, comprehensive datasets and the multicentre design.

\section{Conclusions}

We have demonstrated that neither the ABO blood group nor the RhD status is associated with mortality or morbidity in a mixed critically ill patient population. In sensitivity analyses, the ABO blood group and the RhD status did not add predictive value to the SAPS 3, and in five different subgroup analyses there were no differences in mortality between non-0 blood groups and 
blood group $\mathrm{O}$ or between the RhD status groups. Furthermore, we did not find any differences regarding the number of transfused patients between the ABO blood groups or between the RhD status groups.

\section{Abbreviations}

ARDS: acute respiratory distress syndrome

Covid-19: Coronavirus disease 2019

DAF: Days alive and free of

ICU: Intensive care unit

SAPS 3: Simplified Acute Physiology score 3

SARS-CoV-2: severe acute respiratory syndrome coronavirus-2

SOFA: Sequential Organ Failure Assessment score

vWF: von Willebrand factor

\section{Declarations}

\section{Ethics approval and consent to participate}

The study was approved by the National Ethical Review Board, Lund, Sweden, with registration numbers 2014/916 and $2018 / 866$. As the study was exclusively observational the Board waived the requirement for informed consent. All authors confirm that the study was performed in accordance with the Declaration of Helsinki.

\section{Consent for publication}

Not applicable.

\section{Methods and availability of data and materials}

The datasets used and/or analysed during the current study are available from the corresponding author on reasonable request.

\section{Competing interests}

The authors declare that they have no competing interests.

\section{Funding}

Skåne University Hospital Research Funds, the Skåne County Council's Research. AF: Governmental funding of clinical research within the NHS (National Health Service 2019:YF0053.

\section{Authors' contributions}


TK was the originator of the study, designed the study, constructed the database, performed the statistical analyses and was responsible for the ethical approval. AF was statistical consultant and $\mathrm{MJ}$ assisted in retrieving data from the laboratory information system. All authors contributed to the interpretation of the data. $\mathrm{CN}$ did the main literature search and MB assisted. TK and $\mathrm{CN}$ wrote the first version of the manuscript. All authors revised the manuscript critically, gave final approval of the version to be published and agreed to be accountable for all aspects of the work

\section{Acknowledgements}

The authors would like to acknowledge assistant nurse and data technician Caroline Mårdh for providing comprehensive datasets from the local quality registry PASIVA.

\section{References}

1. Cooling L. Blood Groups in Infection and Host Susceptibility. Clin Microbiol Rev. 2015;28(3):801-70.

2. Anstee DJ. The relationship between blood groups and disease. Blood. 2010;115(23):4635-43.

3. Abegaz SB. Human ABO blood groups and their associations with different diseases. Biomed Res Int. 2021:6629060.

4. Chen Z, Yang SH, Xu H, Li JJ. ABO blood group system and the coronary artery disease: an updated systematic review and meta-analysis. Sci Rep. 2016;623250.

5. Dentali F, Sironi AP, Ageno W, Bonfanti C, Crestani S, Frattini F et al. Relationship between ABO blood group and hemorrhage: a systematic literature review and meta-analysis. Semin Thromb Hemost. 2013;39(01):72-82.

6. Goel R, Bloch EM, Pirenne F, Al-Riyami AZ, Crowe E, Dau L et al. ABO blood group and COVID-19: a review on behalf of the ISBT COVID-19 working group. Vox Sang. 2021;116(8):849-61.

7. Franchini M, Cruciani M, Mengoli C, Marano G, Candura F, Lopez N et al. ABO blood group and COVID-19: an updated systematic literature review and meta-analysis. Blood Transfus. 2021;19(4):317-26.

8. Kim Y, Latz CA, DeCarlo CS, Lee S, Png CM, Kibrik P et al. Relationship between Blood Type and Outcomes Following COVID19 Infection. Semin Vasc Surg. 2021;34(3):125-131.

9. Griffin RL, Jansen JO, Bosarge PL, Marques MB, Kerby JD. The association between ABO blood type and mortality among severely injured trauma patients. Shock. 2020;54(2):205-8.

10. Hamsen U, Nohl A, Baumann A, Lefering R, Boutakmant L, Waydhas $C$ et al. The influence of ABO blood group on mortality in major trauma. Orthop Rev (Pavia). 2019;11(4):8214

11. Hasegawa D, Nishida K, Kawaji T, Hara Y, Shimomura Y, Moriyama K et al. Impact of blood type O on mortality of sepsis patients: a multicenter retrospective observational study. Diagnostics. 2020;10(10):826.

12. Itenov TS, Sessler DI, Khanna AK, Ostrowski SR, Johansson PI, Erikstrup C et al. ABO blood types and sepsis mortality. Ann Intensive Care. 2021;11(1):1-9.

13. Reilly JP, Anderson BJ, Mangalmurti NS, Nguyen TD, Holena DN, Wu Q et al. The ABO histo-blood group and AKI in critically ill patients with trauma or sepsis. Clin J Am Soc Nephrol. 2015;10(11):1911-20.

14. Reilly JP, Meyer NJ, Shashaty MG, Anderson BJ, Ittner C, Dunn TG et al. The ABO Histo-Blood Group, endothelial activation, and acute respiratory distress syndrome risk in critical illness. J Clin Invest. 2021;131(1):e139700.

15. Reilly JP, Meyer NJ, Shashaty MGS, Feng R, Lanken PN, Gallop R et al. ABO blood type A is associated with increased risk of ARDS in whites following both major trauma and severe sepsis. Chest. 2014;145(4):753-61.

16. Rezoagli E, Gatti S, Villa S, Villa G, Muttini S, Rossi F et al. ABO blood types and major outcomes in patients with acute hypoxaemic respiratory failure: A multicenter retrospective cohort study. PLoS One. 2018;13(10):e0206403.

17. Takayama W, Endo A, Koguchi H, Sugimoto M, Murata K, Otomo Y. The impact of blood type O on mortality of severe trauma patients: a retrospective observational study. Critical Care. 2018;22(1):1-7.

18. Takayama W, Endo A, Murata K, Hoshino K, Kim S, Shinozaki H et al. The impact of blood type on the mortality of patients with severe abdominal trauma: a multicenter observational study. Sci Rep. 2021;11(1):1-8. 
19. Tutunjian AM, Arabian SS, Paolino J, Wolfe ES, Mahoney EJ, Hojman HM et al. ABO blood groups do not predict progression of traumatic intracranial hemorrhage. J Clin Neurosci. 2021;90:(345-50.

20. Pendu JL, Breiman A, Rocher J, Dion M, Ruvoën-Clouet N. ABO blood types and COVID-19: spurious, anecdotal, or truly important relationships? A reasoned review of available data. Viruses. 2021;13(2):160.

21. Slade R, Alikhan R, Wise MP, Germain L, Stanworth S, Morgan M. Impact of blood group on survival following critical illness: a single-centre retrospective observational study. BMJ Open Respir Res. 2019;6(1):e000426.

22. Vandenbroucke JP, von Elm E, Altman DG, Gøtzsche PC, Mulrow CD, Pocock SJ et al. Strengthening the Reporting of Observational Studies in Epidemiology (STROBE): Explanation and elaboration. Int J Surg. 2014;12(12):1500-24.

23. Russell JA, Lee T, Singer J, De Backer D, Annane D. Days alive and free as an alternative to a mortality outcome in pivotal vasopressor and septic shock trials. J Crit Care. 2018;47(333-7.

24. Dahlen T, Clements M, Zhao J, Olsson ML, Edgren G. An agnostic study of associations between ABO and RhD blood group and phenome-wide disease risk. Elife. 2021;10:e65658.

\section{Figures}

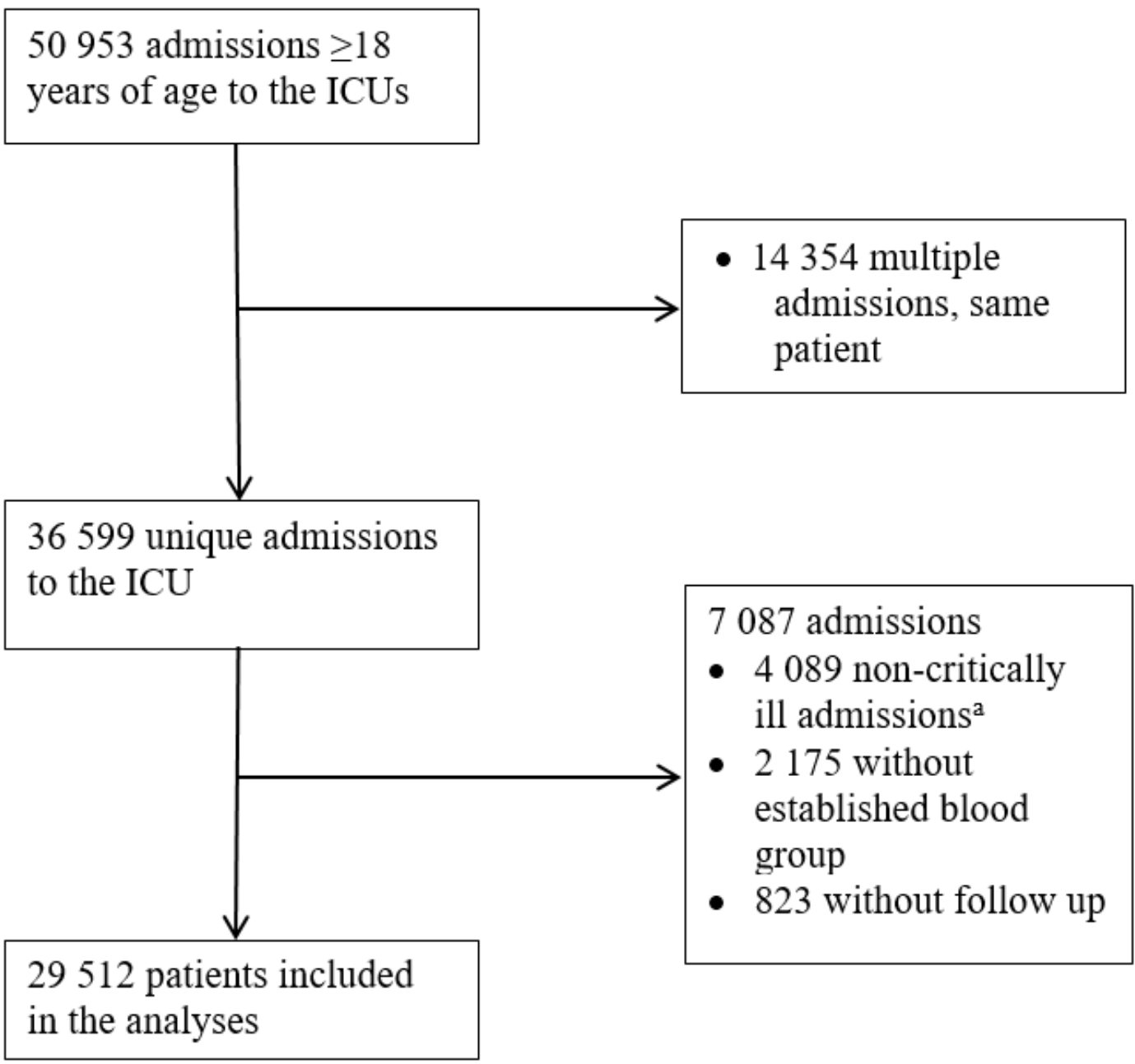

\section{Figure 1}

Consort diagram.

a For example, patients admitted after elective surgery and patients admitted due to hospital shortage of general ward beds. 


\section{Supplementary Files}

This is a list of supplementary files associated with this preprint. Click to download.

- Additionalfile1.docx 